\title{
C-peptide exhibits a late induction effect on matrix metallopeptidase-9 in high glucose-stimulated rat mesangial cells
}

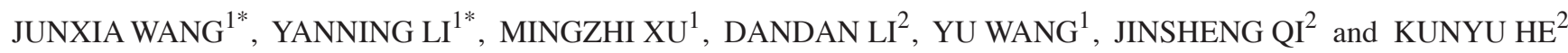 \\ ${ }^{1}$ Department of Molecular Biology, Hebei Key Lab of Laboratory Animal Science; ${ }^{2}$ Department of Biochemistry, \\ Hebei Key Laboratory of Medical Biotechnology, Hebei Medical University, Shijiazhuang, Hebei 050017, P.R. China
}

Received July 9, 2015; Accepted September 6, 2016

DOI: $10.3892 /$ etm.2016.3873

\begin{abstract}
Insufficient matrix metalloproteinase (MMP)-9 and MMP-2 is considered to be a contributor of extracellular matrix (ECM) accumulation in diabetic nephropathy (DN). C-peptide can reverse fibrosis, thus exerting a beneficial effect on DN. Whether C-peptide induces MMP-9 and MMP-2 to reverse ECM accumulation is not clear. In the present study, in order to determine ECM metabolism, rat mesangial cells were treated with high glucose (HG) and C-peptide intervention, then the early and late effects of C-peptide on HG-affected MMP-9 and MMP-2 were evaluated. Firstly, it was confirmed that HG mainly suppressed MMP-9 expression levels. Furthermore, C-peptide treatment induced MMP-9 expression at $6 \mathrm{~h}$ and suppressed it at $24 \mathrm{~h}$, revealing the early dual effects of C-peptide on MMP-9 expression. Subsequently, significant increase in MMP-9 expression at 72, 96 and $120 \mathrm{~h} \mathrm{C}$-peptide treatment was observed. These changes in MMP-9 protein content confirmed its expression changes following late C-peptide treatment. Furthermore, at 96 and 120 h C-peptide treatment reversed the HG-inhibited MMP-9 secretion, further indicating the late induction effect of C-peptide on MMP-9. The present results demonstrated that C-peptide exerted a late induction effect on MMP-9 in HG-stimulated rat mesangial cells, which may be associated with the underlying mechanism of C-peptide's reversal effects on DN.
\end{abstract}

\section{Introduction}

Diabetic nephropathy (DN), the leading cause of end stage renal disease, is the major cause of mortality in type 1 diabetes

Correspondence to: Ms. Yanning Li, Department of Molecular Biology, Hebei Key Lab of Laboratory Animal Science, Hebei Medical University, 361 East Zhongshan Road, Shijiazhuang, Hebei 050017, P.R. China

E-mail: liyanning1981@126.com

*Contributed equally

Key words: C-peptide, matrix metalloproteinase-9, matrix metalloproteinase-2, extracellular matrix, diabetic nephropathy mellitus (DM) and the second most severe complications in type 2 diabetes $(1,2)$. Deposition of extracellular matrix $(\mathrm{ECM})$ in mesangial areas is a feature of $\mathrm{DN}$, and mesangial cells have been proposed to be the determinant of ECM accumulation $(3,4)$. However, the mechanism underlying ECM accumulation in DN is not fully clarified.

Matrix metalloproteinases (MMPs) are a family of zinc-dependent endopeptidases that can degrade numerous types of ECM components (5,6). Among others, MMP-2 basally expresses while MMP-9 is an inducible enzyme, both of which primarily degrade types-I and -IV collagen and laminin, major components of ECM (7-9). Generally, MMP-2 and MMP-9 are involved in tumor metastasis $(6,10)$. Furthermore, it has been shown that insufficient MMP-2 and MMP-9 may be a contributor of ECM accumulation in DN (11). However, the expression levels of MMP-2 and MMP-9 in DN remain controversial, and even short- and long-term hyperglycemia may exert differential effects $(8,12-14)$. As an inducible enzyme, MMP-9 may be more easily affected in patients with DN (15). Therefore, the changes of MMP-2, and particularly MMP-9, for high glucose (HG) stimulation require clarification.

$\mathrm{C}$-peptide is the linker between the A-chain and B-chain of insulin. Lack of C-peptide along with insulin is the primary feature of type $1 \mathrm{DM}$ and late stage of type $2 \mathrm{DM}$ (16). C-peptide has been found to have unique beneficial effects on DN, attenuating glomerular and tubular injury (17-19). Physiological concentration of $\mathrm{C}$-peptide can reverse the fibrosis of glomerular and recover renal function in DN (20-22). Various mechanisms have been reported for the protective effects of C-peptide, such as binding to its receptor on the cell membrane, transporting into the cytoplasm and nucleus, and interacting with functional proteins to exhibit its effect (23-25). In a prior study, we observed that C-peptide could dynamically localize in the nucleus to serve its functions in HG-stimulated mesangial cells, which provided an impetus for further clarifying the intrinsic mechanism of its unique reversal effect on DN (26). Although it has been reported that C-peptide exerted little effect on MMP-2 in diabetic rats (22), the short- and long-term effects of C-peptide on MMP-9 and MMP-2 in HG-treated mesangial cells remains unknown.

In the present study, rat mesangial cells were cultured to investigate the short- and long-term effects of C-peptide on HG-affected MMP-9 and MMP-2 expression levels. After mesangial cells were treated, MMP-9 and MMP-2 mRNA 
expression levels, MMP-9 protein content and secretion were evaluated using reverse transcription-quantitative polymerase chain reaction (RT-qPCR), western blot and enzyme-linked immunosorbent assay (ELISA) analyses.

\section{Materials and methods}

Cells and treatment. The rat mesangial cell line (HBZY-1) was obtained from China Center for Type Culture Collection (Wuhan, China) and cultured in Dulbecco's modified Eagle's medium (DMEM; Thermo Fisher Scientific Co., Ltd., Shanghai, China) containing $5 \mathrm{mM}$ glucose and $10 \%$ fetal bovine serum (Thermo Fisher Scientific Co., Ltd.). Cells were cultured in 20, 25, 30 and 35 mM HG (Thermo Fisher Scientific Co., Ltd.) or control (5 mM) glucose for $24 \mathrm{~h}$, then treated with $30 \mathrm{mM} \mathrm{HG}$ for 3, 6, 12, 24, 48 and $72 \mathrm{~h}$. MMP-9 and MMP-2 mRNA expression levels were subsequently evaluated. Subsequently, 0.1, 0.3, 0.5, 0.7 and 0.9 nM C-peptide (Shanghai Taishi Biotechnology Co., Ltd., Shanghai, China) was used to treat the HG-stimulated mesangial cells for 3, 6 , 12 and $24 \mathrm{~h}$, then $0.7 \mathrm{nM} \mathrm{C}$-peptide treatment expanded to $120 \mathrm{~h}$, the MMP-9 and MMP-2 mRNA expression levels were evaluated. Furthermore, MMP-9 protein content was evaluated for 6, 72, 96 and $120 \mathrm{~h} \mathrm{C}$-peptide treatment. In addition, the MMP-9 secretion for HG and C-peptide treatments were detected. Low glucose (LG, $5 \mathrm{mM}$ ) was used as control.

RT-qPCR. The MMP-9 and MMP-2 transcription was evaluated by RT-qPCR. Total RNA was isolated using TRIzol reagent (Takara Bio, Inc., Otsu, Japan) and reverse transcribed into cDNA using RevertAid First Strand cDNA synthesis Kit (Fermentas; Thermo Fisher Scientific, Inc., Waltham, MA, USA), followed by PCR amplification using the specific primers (Sangon Biotech Co., Ltd., Shanghai, China). Rat MMP-9 forward primer, 5'-AAACCCTGCGTATTTCCA TTCATC-3', and reverse primer, 5'-CACATCTCTCCTGCC GAGTTGC-3' with 185 bp product; MMP-2 forward primer, 5'-TGGAAGCATCAAATCGGACTG-3', and reverse primer, 5'-CCACCCTCTTAAATCTGAAATCAC-3' with 186 bp product. A Rotor-Gene 3000 system (Corbett Life Science; Qiagen, Shenzhen, China) was used to perform the PCR reaction, using aSYBR Premix Ex Taq II (RR82LR; Takara Biotechnology Co., Ltd., Dalian, China) and analyze the data. Actin primers were used as an internal standard.

Western blot analysis. The protein content of MMP-9 was detected using the protocol previously described, using an anti-MMP-9 antibody (1:500; \#3852; Cell Signaling Technology, Inc., Danvers, MA, USA) (27), and the horseradish peroxidase-labeled secondary antibody (1:5,000; 074-1506; Kirkegaard \& Perry Lab, Inc., Gaithersburg, MA, USA). Band intensity was quantified and calculated. Actin was routinely served as a loading control (1:1,000; \#4967; Cell Signaling Technology, Inc., Danvers, MA, USA).

ELISA. Following treatment, the culture medium was collected to analyze the MMP-9 secretion by ELISA (H146-4; Nanjing Jiancheng Bioengineering Institute, Nanjing, China), according to the manufacturer's instructions. Standards and samples were added to wells of the plate and incubated for
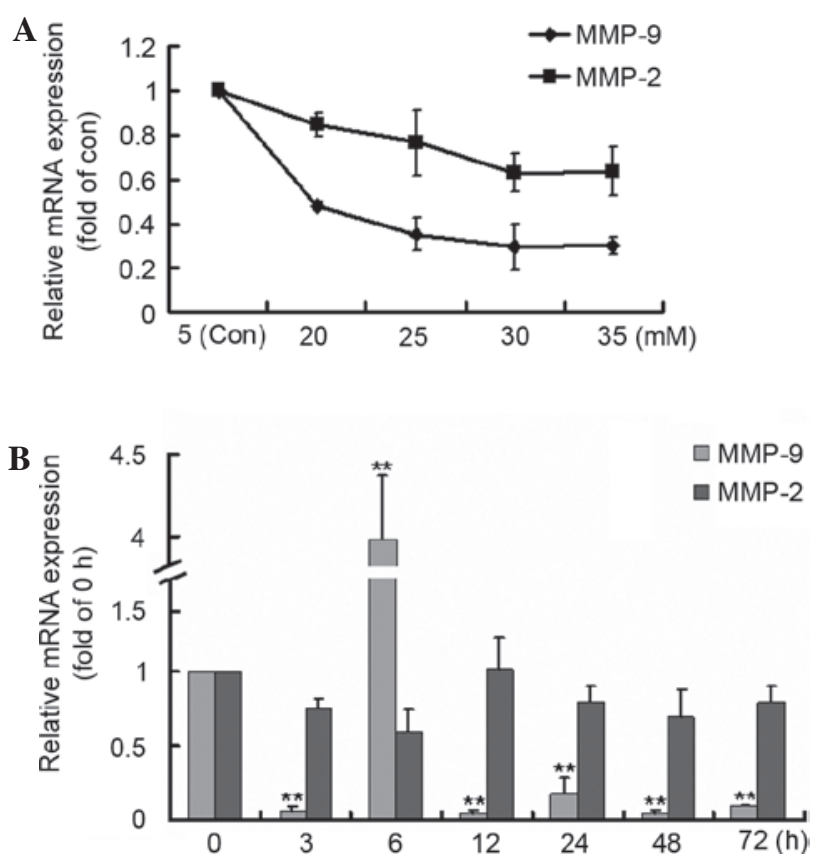

Figure 1. Effects of high glucose (HG) on MMP-9 and MMP-2 mRNA expression levels. The mRNA expression levels of MMP-9 and MMP-2 were detected using reverse transcription-quantitative polymerase chain reaction. (A) After the cells were stimulated with different concentrations of HG, MMP-9 and MMP-2 expression decreased, most markedly at $30 \mathrm{mM}$ HG treatment. (B) For 30 mM HG treatment, MMP-9 expression decreased significantly, except for its sharp increase at $6 \mathrm{~h}$, while MMP-2 expression showed no significant changes. ${ }^{* *} \mathrm{P}<0.01$ vs. 0 h group. Experiments were repeated three times. MMP, matrix metalloproteinase.

$1 \mathrm{~h}$. After the wells were washed with the ELISA wash buffer, the conjugated antibody was added and incubated for $1 \mathrm{~h}$. Then the wells were washed with the ELISA wash buffer. The substrate was added in the wells and incubated for $15 \mathrm{~min}$. The stop solution was added and absorption was measured using an ELISA reader at $450 \mathrm{~nm}$ (Multiskan Spectrum: Thermo Fisher Scientific, Inc.). All tests were performed in duplicate.

Statistical analysis. Statistical analysis of the data was performed using SPSS 17.0 software (SPSS, Inc., Chicago, IL, USA). Comparisons between two groups were performed using Student's $t$-test. All values are presented as the mean \pm standard deviation. $\mathrm{P}<0.05$ were considered to indicate a statistically significant difference.

\section{Results}

Early dual effects of C-peptide on MMP-9 expression in $H G$-treated mesangial cells. The concentration and time-dependent effects of HG on MMP-9 and MMP-2 expression levels were detected. Both MMP-9 and MMP-2 expression levels decreased following HG stimulation, most markedly at $30 \mathrm{mM} \mathrm{HG}$ (Fig. 1A). Then at $30 \mathrm{mM} \mathrm{HG}$ incubation, MMP-9 expression decreased significantly compared with the $0 \mathrm{~h}$ group, except for an increase at $6 \mathrm{~h}$; however, MMP-2 expression showed no significant changes (Fig. 1B). The results confirmed that HG suppressed MMP-9 expression in mesangial cells.

After pretreatment with $\mathrm{HG}$ for $24 \mathrm{~h}$, the early effects of C-peptide on MMP-9 and MMP-2 expression were investigated. 

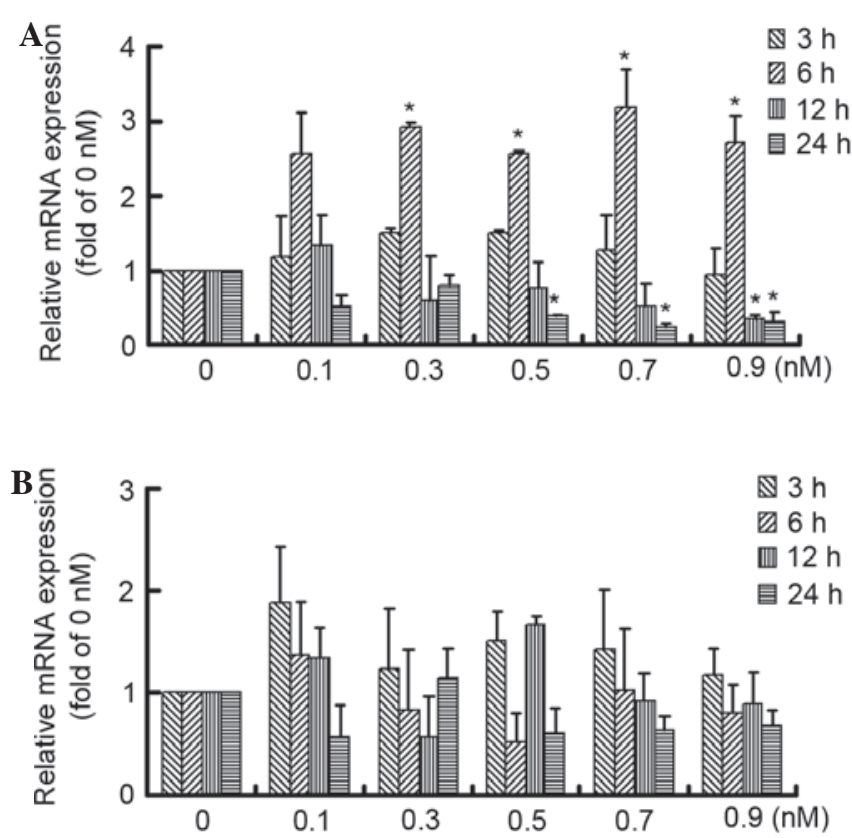

Figure 2. Early effects of C-peptide on high glucose (HG)-affected matrix metalloproteinase. (MMP)-9 and MMP-2 mRNA expression levels. After the cells were pretreated with $\mathrm{HG}$ for $24 \mathrm{~h}$, and incubated with different concentrations of C-peptide for different priods, the mRNA expression levels of MMP-9 and MMP-2 were detected by reverse transcription-quantitative polymerase chain reaction. (A) MMP-9 expression could be induced at $6 \mathrm{~h}$, but significantly inhibited along with treatment time, most markedly at $24 \mathrm{~h}$, with similar effects of $0.5,0.7$ and $0.9 \mathrm{nM} \mathrm{C}$-peptide treatment. (B) MMP-2 expression showed no significant differences among groups. "P<0.05 vs. $0 \mathrm{nM}$ group. Experiments were repeated three times.

Although C-peptide treatment induced an increase in MMP-9 expression at $6 \mathrm{~h}$, MMP-9 expression decreased over time, particularly at $24 \mathrm{~h}$. Furthermore, $0.5,0.7$ and $0.9 \mathrm{nM}$ C-peptide produced a similar effect on MMP-9 expression (Fig. 2A). However, no significant difference in MMP-2 expression was observed among groups (Fig. 2B). The results showed that C-peptide exhibited an early dual effect on MMP-9 expression within $24 \mathrm{~h}$ treatment in HG-treated mesangial cells.

Late induction effect of C-peptide on MMP-9 in HG-treated mesangial cells. The treatment time was expanded to $120 \mathrm{~h}$, and the late effects of C-peptide on MMP-9 and MMP-2 expression levels were investigated. It was found that MMP-9 expression increased markedly between cells treated for 72 and $96 \mathrm{~h}$, although the early changes were inconsistent and not significant (Fig. 3A). However, MMP-2 expression was disordered, but significantly decreased following 96 and $120 \mathrm{~h}$ of treatment (Fig. 3B).

Then, the late induction effect of C-peptide on MMP-9 expression was further verified by evaluation of its protein content (Fig. 4). Compared with that in the LG group, the MMP-9 protein content decreased in the HG group. The HG-inhibited MMP-9 protein content was significantly induced by 72, 96 and $120 \mathrm{~h}$ of C-peptide treatment. The results suggest that $\mathrm{C}$-peptide had a late induction effect on MMP-9 in HG-treated mesangial cells.

Late reversal effect of C-peptide on HG-suppressed MMP-9 secretion. After treatment, the culture medium was collected
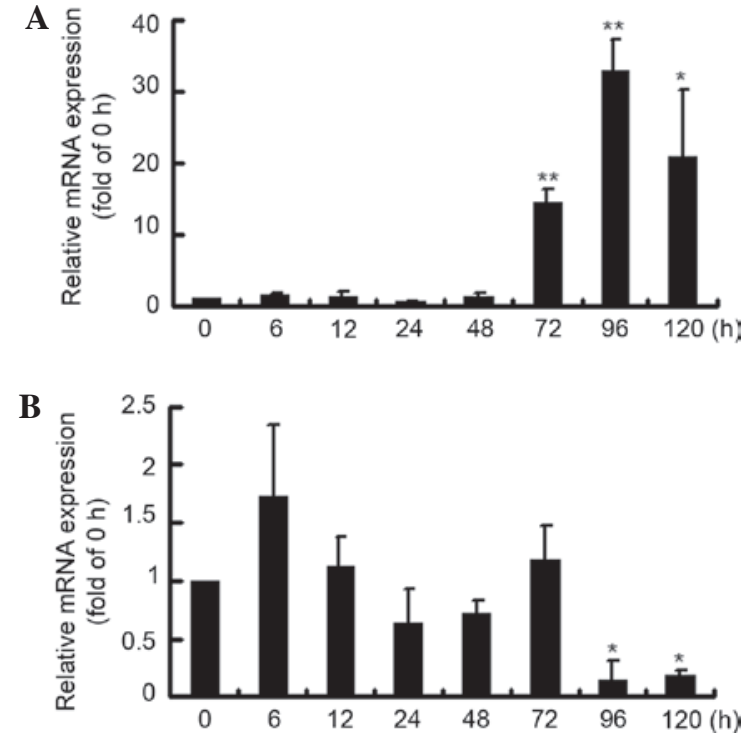

Figure 3. Late effects of C-peptide on high glucose (HG)-affected matrix metalloproteinase. (MMP)-9 and MMP-2 mRNA expression. After the cells were pretreated with $\mathrm{HG}$ for $24 \mathrm{~h}$, and incubated with $0.7 \mathrm{nM} \mathrm{C}$-peptide for different time, the mRNA expression levels of MMP-9 and MMP-2 were detected by reverse transcription-quantitative polymerase chain reaction. (A) MMP-9 expression increased significantly at 72,96 and $120 \mathrm{~h}$ treatment. (B) MMP-2 expression decreased at 96 and 120 h treatment. ${ }^{* *} \mathrm{P}<0.01$ or ${ }^{*} \mathrm{P}<0.05$ vs. 0 h group. Experiments were repeated three times.

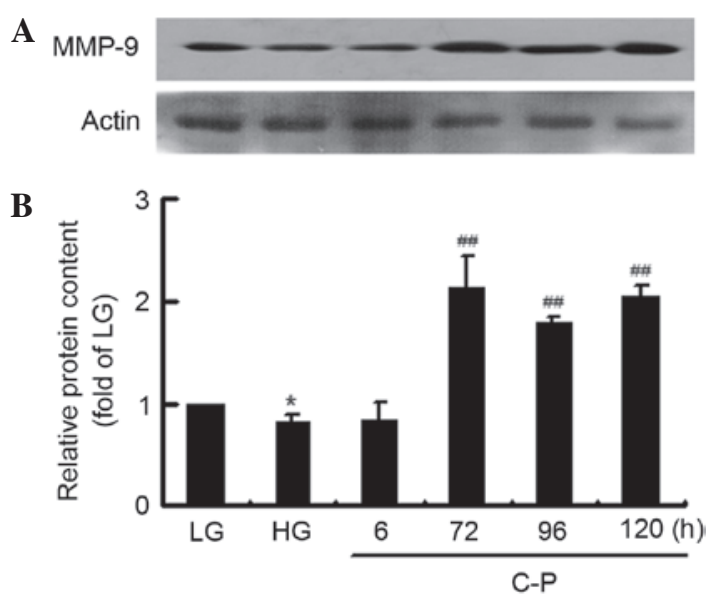

Figure 4. Late effects of C-P on HG-suppressed MMP-9 protein content. (A) After the cells were pretreated with $\mathrm{HG}$ for $24 \mathrm{~h}$, and incubated with $0.7 \mathrm{nM}$ C-P for different time, MMP-9 protein content was detected by western blot analysis. (B) Although decreased for HG incubation, the MMP-9 protein content increased significantly for 72, 96 and $120 \mathrm{~h} \mathrm{C}$-peptide treatment. ${ }^{*} \mathrm{P}<0.05$ vs. LG group; ${ }^{\# \#} \mathrm{P}<0.01$ vs. HG group. Experiments were repeated three times. MMP, matrix metalloproteinase; LG, low glucose; HG, high glucose; C-P, C-peptide.

for detection of MMP-9 secretion. The HG-suppressed MMP-9 secretion from mesangial cells was initially verified. MMP-9 secretion was found to be suppressed by 25 and $30 \mathrm{mM} \mathrm{HG}$ treatment (Fig. 5A). Furthermore, after $30 \mathrm{mM} \mathrm{HG}$ incubation, the MMP-9 secretion time-dependently decreased, with a significant difference at 24, 48 and $72 \mathrm{~h}$ (Fig. 5B).

Thus, the effects of C-peptide on the HG-suppressed MMP-9 secretion were investigated (Fig. 5C). Compared with the LG group, the MMP-9 secretion was significantly inhibited by HG incubation. Furthermore, HG-suppressed 

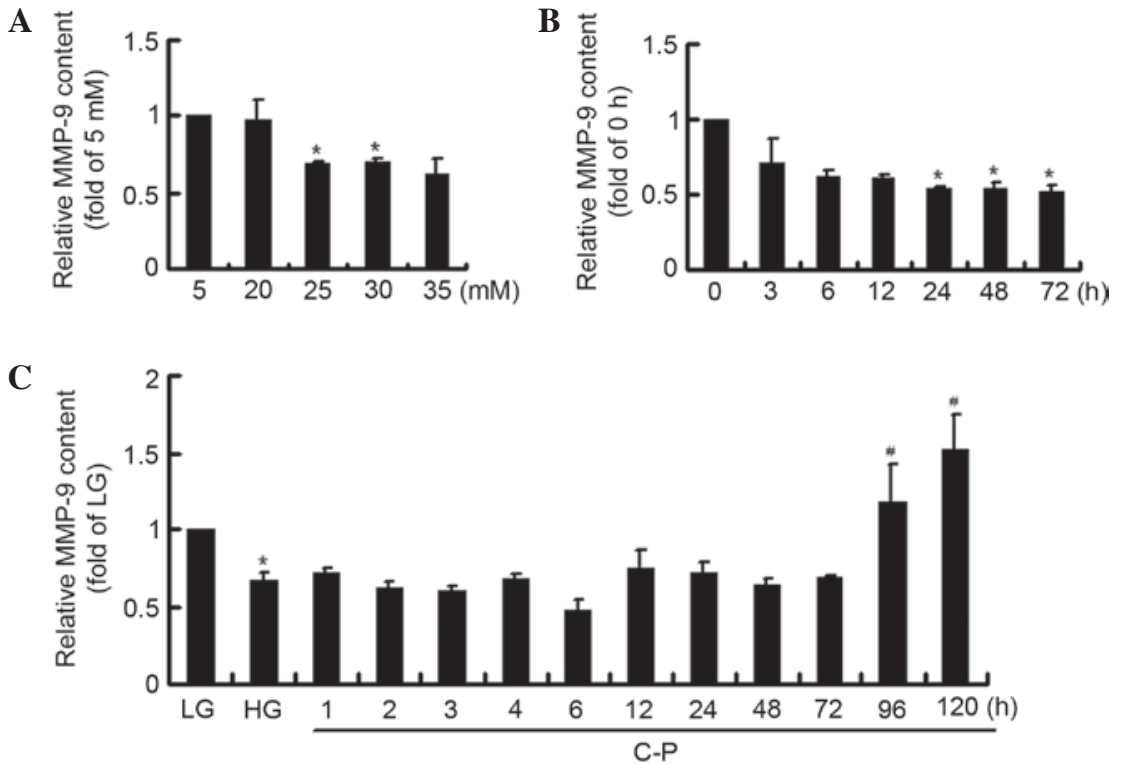

Figure 5. Effects of C-P on HG-suppressed MMP-9 secretion. After treatment, the culture medium was collected for enzyme-linked immunosorbent assay detection of MMP-9 secretion. (A) MMP-9 secretion was significantly inhibited by 25 and $30 \mathrm{mM} \mathrm{HG}$ incubation for 24 h. "P<0.05 vs. 5 mM group. (B) MMP-9 secretion was significantly suppressed by $30 \mathrm{mM} \mathrm{HG}$ incubation. "P<0.05 vs. 0 h group. (C) HG inhibited MMP-9 secretion was significantly increased for $0.7 \mathrm{nM} \mathrm{C}-\mathrm{P}$ treatment at 96 and $120 \mathrm{~h}$. "P $<0.05$ vs. LG group; ${ }^{"} \mathrm{P}<0.05$ vs. HG group. Experiments were repeated three times. MMP, matrix metalloproteinase; LG, low glucose; HG, high glucose; C-P, C-peptide.

MMP-9 secretion was significantly increased at 96 and $120 \mathrm{~h}$ C-peptide treatment, although no evident effect was observed earlier than this. The results demonstrated that $\mathrm{C}$-peptide had a late reversal effect on the HG-suppressed MMP-9 secretion.

\section{Discussion}

Although C-peptide has reversal effects on the fibrosis of glomerular in DN (18-22), the underlying mechanism is not clarified. Insufficient MMP-2 and MMP-9 is considered to be a contributor of ECM accumulation (11). Whether C-peptide regulates MMP-2 and MMP-9 to reverse fibrosis is unclear. In the present study, we found that $\mathrm{C}$-peptide exhibited a late induction effect on MMP-9 in HG-stimulated rat mesangial cells, which may represent the underlying mechanism of C-peptide's reversal effects on DN.

Basal MMP-2 and inducible MMP-9 primarily degrade collagen and laminin, major components of ECM (28). Although insufficient MMP-2 and MMP-9 may lead to ECM accumulation, the expression levels of MMP-2 and MMP-9 in DN remain controversial (29). In the present study, marked changes of MMP-2 expression were not observed for $\mathrm{HG}$ stimulation. The MMP-9 expression was markedly inhibited by $\mathrm{HG}$ treatment, with the exception of a sharp increase at 6 h. Furthermore, HG-inhibited MMP-9 was verified by its secretion detection. The results revealed that as an inducible enzyme, MMP-9 was more susceptible to be affected by HG, which predominantly inhibited MMP-9 expression, indicating that MMP-9 insufficiency may be a contributing factor of ECM accumulation in DN.

C-peptide has reversal effects on the fibrosis of glomerular in DN (30). Although a number of mechanisms have been reported for the protective effects of C-peptide (23-25), they are not specific to DN and to not fully explain the anti-fibrosis effects of $\mathrm{C}$-peptide. It has been reported that $\mathrm{C}$-peptide exerted little effect on MMP-2, which is basally expressed and was hardly affected by HG (19). On the other hand, whether C-peptide induces MMP-9 to reverse ECM accumulation is unknown. In the present study, the short- and long-term effects of C-peptide on MMP-9 and MMP-2 in HG-treated mesangial cells were investigated.

Firstly, the early effects of C-peptide on MMP-9 and MMP-2 expression levels at $24 \mathrm{~h}$ were detected. The MMP-2 expression showed no significant changes, consistent with previous results (22). Physiological concentrations of C-peptide inhibited MMP-9 expression at $24 \mathrm{~h}$ treatment, except for a sharp increase at $6 \mathrm{~h}$, revealing the early dual effects of C-peptide on MMP-9 expression. Next, the treatment time was expanded to $120 \mathrm{~h}$ to investigate the late effects of C-peptide on MMP-9 and MMP-2 expression levels. Notably, it was found that MMP-9 expression was markedly induced, while MMP-2 expression was inhibited. In addition, the changes of MMP-9 protein content confirmed the late induction effect of C-peptide on MMP-9 expression.

Furthermore, ELISA results showed that C-peptide had a significant late reversal effect on the HG-inhibited MMP-9 secretion, although the early effect was unchanged. The decreased MMP-9 secretion in response to HG stimulation and unchanged MMP-9 secretion for short-period C-peptide treatment indicated that the sharp increases in MMP-9 mRNA expression in response to $\mathrm{HG}$ and $\mathrm{C}$-peptide at $6 \mathrm{~h}$ may be due to the inducibility of MMP-9 mRNA.

In conclusion, the results demonstrated that C-peptide exhibited a late induction effect on MMP-9 in HG-stimulated rat mesangial cells, which may be associated with the underlying mechanism of C-peptide's reversal effects on DN.

\section{Acknowledgements}

This study was supported by Grants from the Major State Basic Research Development Program of China (973 Program; grant 
no. 2012CB518601), the National Natural Science Foundation of China (grant no. 81070658), the Hebei Natural Science Foundation (grant no. H2012206005).

\section{References}

1. Kato $M$ and Natarajan R: Diabetic nephropathy-emerging epigenetic mechanisms. Nat Rev Nephrol 10: 517-530, 2014.

2. Zhang Y, Xiao HQ, Wang Y, Yang ZS, Dai LJ and Xu YC: Differential expression and therapeutic efficacy of microRNA-346 in diabetic nephropathy mice. Exp Ther Med 10: 106-112, 2015.

3. Zhang L, Zhang J, Liu X, Liu S and Tian J: Tribbles 3 regulates the fibrosis cytokine TGF- $\beta 1$ through ERK1/2-MAPK signaling pathway in diabetic nephropathy. J Immunol Res 2014: 240396, 2014.

4. Miller CG, Pozzi A, Zent R and Schwarzbauer JE: Effects of high glucose on integrin activity and fibronectin matrix assembly by mesangial cells. Mol Biol Cell 25: 2342-2350, 2014.

5. Galliera E, Tacchini L and Corsi Romanelli MM: Matrix metalloproteinases as biomarkers of disease: Updates and new insights. Clin Chem Lab Med 53: 349-355, 2015.

6. Tauro M, McGuire J and Lynch CC: New approaches to selectively target cancer-associated matrix metalloproteinase activity. Cancer Metastasis Rev 33: 1043-1057, 2014.

7. Piperi $\mathrm{C}$ and Papavassiliou AG: Molecular mechanisms regulating matrix metalloproteinases. Curr Top Med Chem 12 1095-1112, 2012

8. Fukami K, Yamagishi S, Coughlan MT, Harcourt BE, Kantharidis P, Thallas-Bonke V, Okuda S, Cooper ME and Forbes JM: Ramipril inhibits AGE-RAGE-induced matrix metalloproteinase-2 activation in experimental diabetic nephropathy. Diabetol Metab Syndr 6: 86, 2014.

9. Zhong Y, Zhang X, Cai X, Wang K, Chen Y and Deng Y: Puerarin attenuated early diabetic kidney injury through down-regulation of matrix metalloproteinase 9 in streptozotocin-induced diabetic rats. PLoS One 9: e85690, 2014.

10. Lu H, Cao X, Zhang H, Sun G, Fan G, Chen L and Wang S: Imbalance between MMP-2, 9 and TIMP-1 promote the invasion and metastasis of renal cell carcinoma via SKP2 signaling pathways. Tumour Biol 35: 9807-9813, 2014.

11. Sun H, Ge N, Shao M, Cheng X, Li Y, Li S and Shen J: Lumbrokinase attenuates diabetic nephropathy through regulating extracellular matrix degradation in Streptozotocin-induced diabetic rats. Diabetes Res Clin Pract 100: 85-95, 2013.

12. Thrailkill KM, Clay Bunn R and Fowlkes JL: Matrix metalloproteinases: Their potential role in the pathogenesis of diabetic nephropathy. Endocrine 35: 1-10, 2009.

13. Lewandowski KC, Banach E, Bieńkiewicz M and Lewiński A Matrix metalloproteinases in type 2 diabetes and non-diabetic controls: Effects of short-term and chronic hyperglycaemia. Arch Med Sci 7: 294-303, 2011.

14. Li SY, Huang PH, Yang AH, Tarng DC, Yang WC, Lin CC, Chen JW, Schmid-Schönbein G and Lin SJ: Matrix metalloproteinase-9 deficiency attenuates diabetic nephropathy by modulation of podocyte functions and dedifferentiation. Kidney Int 86: 358-369, 2014.

15. Potier M, Elliot SJ, Tack I, Lenz O, Striker GE, Striker LJ and Karl M: Expression and regulation of estrogen receptors in mesangial cells: Influence on matrix metalloproteinase-9. J Am Soc Nephrol 12: 241-251, 2001.
16. Bhatt MP, Lim YC, Hwang J, Na S, Kim YM and Ha KS: C-peptide prevents hyperglycemia-induced endothelial apoptosis through inhibition of reactive oxygen species-mediated transglutaminase 2 activation. Diabetes 62: 243-253, 2013.

17. Al-Rasheed NM, Willars GB and Brunskill NJ: C-peptide signals via Galpha i to protect against TNF-alpha-mediated apoptosis of opossum kidney proximal tubular cells. J Am Soc Nephrol 17: 986-995, 2006

18. Hills CE, Brunskill NJ and Squires PE: C-peptide as a therapeutic tool in diabetic nephropathy. Am J Nephrol 31: 389-397, 2010.

19. Wahren J, Kallas A and Sima AA: The clinical potential of C-peptide replacement in type 1 diabetes. Diabetes 61: 761-772, 2012.

20. Samnegård B, Jacobson SH, Jaremko G, Johansson BL and Sjöquist M: Effects of C-peptide on glomerular and renal size and renal function in diabetic rats. Kidney Int 60: 1258-1265, 2001.

21. Huang DY, Richter K, Breidenbach A and Vallon V: Human C-peptide acutely lowers glomerular hyperfiltration and proteinuria in diabetic rats: A dose-response study. Naunyn Schmiedebergs Arch Pharmacol 365: 67-73, 2002.

22. Sun W, Gao X, Zhao X, Cui D and Xia Q: Beneficial effects of C-peptide on renal morphology in diabetic rats. Acta Biochim Biophys Sin (Shanghai) 42: 893-899, 2010.

23. Ishii T, Fukano K, Shimada K, Kamikawa A, Okamatsu-Ogura Y, Terao A, Yoshida T, Saito M and Kimura K: Proinsulin C-peptide activates $\alpha$-enolase: Implications for C-peptide-cell membrane interaction. J Biochem 152: 53-62, 2012.

24. Luppi P, Geng X, Cifarelli V, Drain P and Trucco M: C-peptide is internalized in human endothelial smooth muscle cells via early endosomes. Diabetologia 52: 2218-2228, 2009.

25. Lindahl E, Nyman U, Zaman F, Palmberg C, Cascante A, Shafqat J, Takigawa M, Sävendahl L, Jörnvall H and Joseph B: Proinsulin C-peptide regulates ribosomal RNA expression. J Biol Chem 285: 3462-3469, 2010.

26. Li Y,Zhao M, Li B and Qi J: Dynamic localization and functional implications of C-peptide might for suppression of iNOS in high glucose-stimulated rat mesangial cells. Mol Cell Endocrinol 381: 255-260, 2013.

27. Li Y, Liu D, Liu Y, Li E, Wang H, Liu K and Qi J: Protein nitration promotes inducible nitric oxide synthase transcription mediated by NF- $\kappa$ B in high glucose-stimulated human lens epithelial cells. Mol Cell Endocrinol 370: 78-86, 2013.

28. Lehners A, Lange S, Niemann G, Rosendahl A, Meyer-Schwesinger C, Oh J, Stahl R, Ehmke H, Benndorf R, Klinke A, et al: Myeloperoxidase deficiency ameliorates progression of chronic kidney disease in mice. Am J Physiol Renal Physiol 307: F407-F417, 2014.

29. Kuno Y, Iyoda M, Shibata T, Hirai Y and Akizawa T: Sildenafil, a phosphodiesterase type 5 inhibitor, attenuates diabetic nephropathy in non-insulin-dependent otsuka long-evans tokushima fatty rats. Br J Pharmacol 162: 1389-1400, 2011.

30. Samnegård B, Jacobson SH, Jaremko G, Johansson BL, Ekberg K, Isaksson B, Eriksson L, Wahren J and Sjöquist M: C-peptide prevents glomerular hypertrophy and mesangial matrix expansion in diabetic rats. Nephrol Dial Transplant 20: $532-538,2005$. 